\title{
Multiparametric magnetic resonance imaging of prostate cancer
}

\author{
Sandeep S Hedgire, Tamara N Oei, Shaunagh Mcdermott, Kai Cao, Zena Patel M11, Mukesh G Harisinghani \\ Department of Abdominal Imaging and Intervention, Massachusetts General Hospital 55 Fruit St, Boston, 02114 Massachusetts, USA, \\ 'Department of Radiology, P. D. Hinduja Hospital National Hospital and Medical Research Centre, Mahim, Mumbai, India
}

Correspondence: Dr. Zena Patel M, Department of Radiology, P. D. Hinduja Hospital National Hospital and Medical Research Centre, Mahim, Mumbai - 400 016, India. E-mail: patelzena@hotmail.com

\begin{abstract}
In India, prostate cancer has an incidence rate of 3.9 per 100,000 men and is responsible for $9 \%$ of cancer-related mortality. It is the only malignancy that is diagnosed with an apparently blind technique, i.e., transrectal sextant biopsy. With increasing numbers of high-Tesla magnetic resonance imaging (MRI) equipment being installed in India, the radiologist needs to be cognizant about endorectal MRI and multiparametric imaging for prostate cancer. In this review article, we aim to highlight the utility of multiparamteric $\mathrm{MRI}$ in prostate cancer. It plays a crucial role, mainly in initial staging, restaging, and post-treatment follow-up.
\end{abstract}

Key words: Diffusion-weighted imaging; dynamic contrast-enhanced-magnetic resonance imaging; magnetic resonance imaging; prostate cancer

\section{Introduction}

Prostate cancer is the most commonly diagnosed malignancy in men, with almost one-quarter of males diagnosed with malignancy having cancer of the prostate. The age-adjusted incidence rate in the United States is 156 per 100,000 men per year. ${ }^{[1]}$ In India prostate cancer has an incidence rate of 3.9 per 100,000 men and is responsible for $9 \%$ of all cancer-related mortality. ${ }^{[2,3]}$ It is the only malignancy that is diagnosed with an apparently blind technique, i.e., transrectal sextant biopsy. Magnetic resonance imaging (MRI) plays a crucial role mainly in the initial staging, restaging, and post-treatment follow-up of cases of prostate cancer. The superior soft tissue resolution, multiplanar imaging capability, and technical refinements have established MRI as the most accurate modality for the detection and staging of prostate cancer. ${ }^{[4]}$ The goal of this review is to provide a comprehensive update on advanced

\begin{tabular}{|l|l|}
\hline \multicolumn{2}{|c|}{ Access this article online } \\
\hline Quick Response Code: & Website: \\
& www.ijri.org \\
\hline
\end{tabular}

MRI techniques for improving the detection, staging, and post-treatment follow-up of patients with prostate cancer.

\section{MRI Anatomy of Normal Prostate Gland and The Technique of Endorectal Magnetic Resonance Imaging}

Anatomically, the prostate gland is divided into four parts: The peripheral zone, the transitional zone, the central zone, and the anterior nonglandular fibromuscular stroma. The peripheral zone comprises $70-80 \%$ of the glandular tissue, and $70 \%$ of prostate cancers arise in this zone. ${ }^{[5]}$ The remaining $30 \%$ of cancers occur in the transition zone. On T2-weighted (T2W) images, the central and transitional zones cannot be distinguished and are collectively called the central gland, which is separated from the peripheral zone by a thin pseudocapsule ${ }^{[6]}$ [Figure 1]. On T2W images the peripheral zone shows high signal intensity, which is either equal to or more than that of the fat in the vicinity ${ }^{[7]}[$ Figure 2]. The high signal intensity is attributed to the fluid-filled ductal and acinar components, with age-related increase in the signal intensity. ${ }^{[8-10]}$ Compared with the peripheral zone, the central gland displays a low or heterogenous T2 signal intensity since it contains fewer glandular structures and smooth muscles. The central gland may appear heterogeneous due to the presence of nodules and cysts ${ }^{[1]}$ [Figure 3]. The true capsule, seen as a 
low-intensity rim, is best appreciated on the posterior and posterolateral aspects of the gland [Figure 4]. This capsule is an important imaging landmark in prostate cancer as extracapsular extension (ECE) can upstage the tumor to $\mathrm{T}_{3}$. Neurovascular bundles can usually be seen on axial images at 5 and 7 o'clock positions [Figure 4]. The penetrating branches of the neurovascular bundles to the apex and base of the gland serve as important pathways for extension of the tumor outside the capsule. ${ }^{[7]}$ The seminal vesicles [Figure 5] are seen as elongated fluid-filled structures with thin septae and are seen as low signal intensity on T1-weighted (T1W)

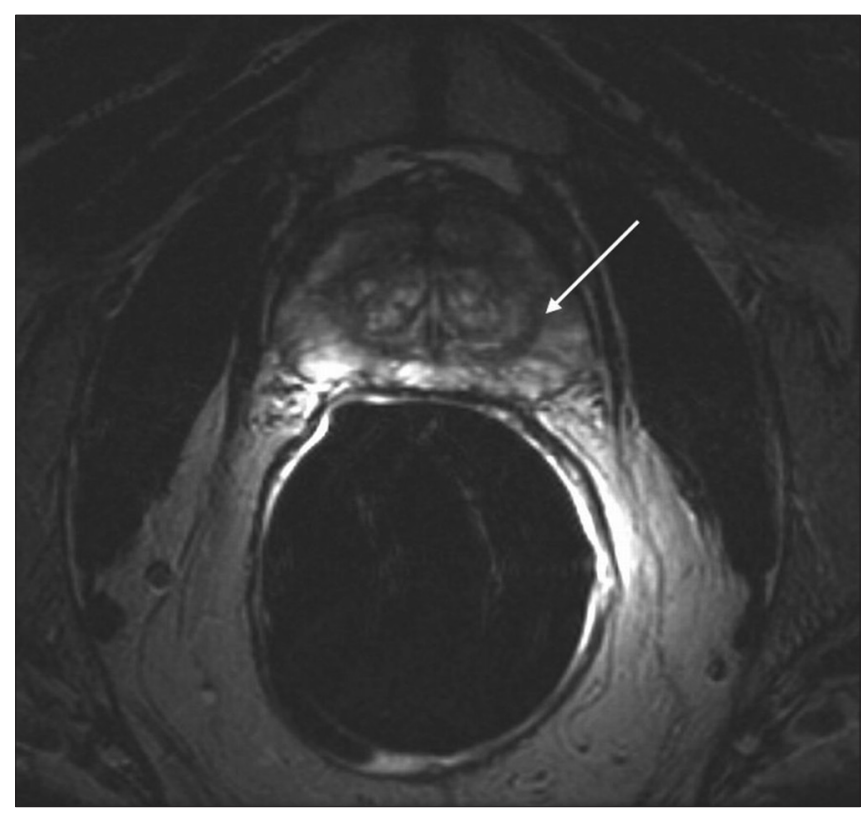

Figure 1: T2-weighted axial images show pseudocapsule (arrow) separating central zone and peripheral zone

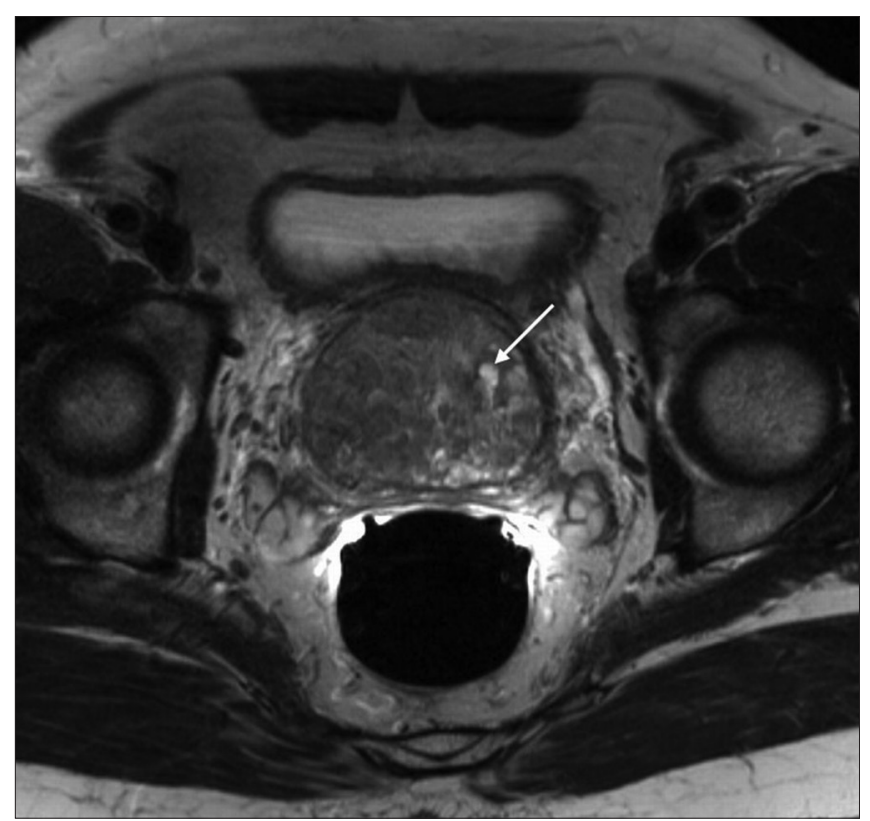

Figure 3: Axial T2-weighted images show heterogenous signal of transitional zone with a cyst (arrow) images and high signal intensity on T2W images. The vas deferens is seen as a tubular structure medial to the seminal vesicles and displays low T1 and T2 signal intensity. ${ }^{[12]}$ The seminal vesicles and the vas are better appreciated on coronal and axial images.

The European Consensus Meeting divided the prostate into a minimum of 16 - and optimally 27 - regions of interest [Figure 6] and suggested that a score of 1-5 to be assigned for each region, with a score of " 1 " denoting that clinically significant disease was highly unlikely to be present and a score of " 5 " denoting that clinically significant

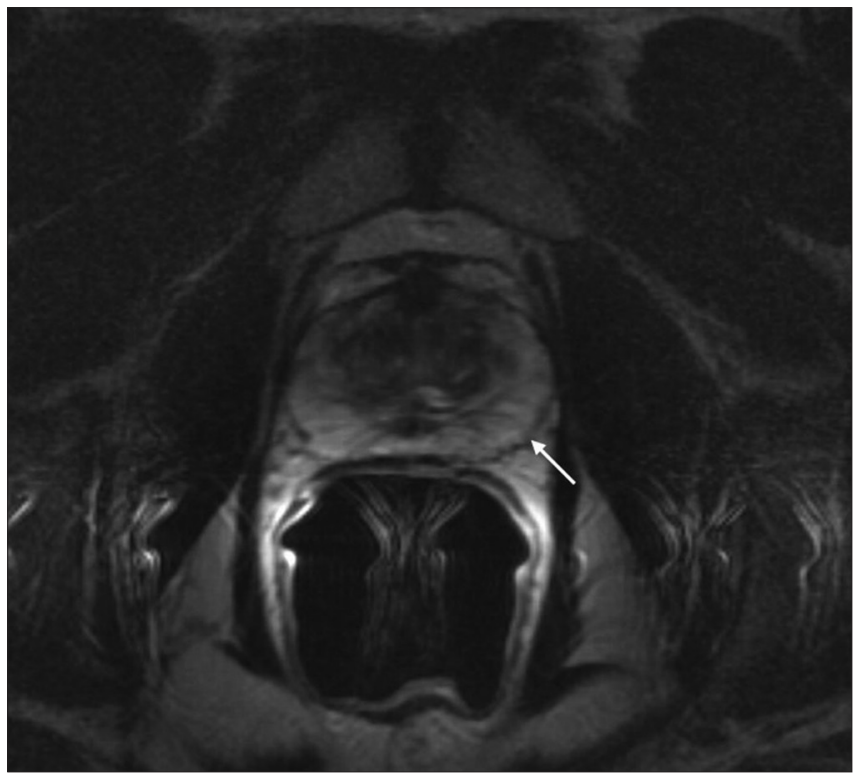

Figure 2: T2-weighted axial image show hyperintense signal of normal peripheral zone (arrow)

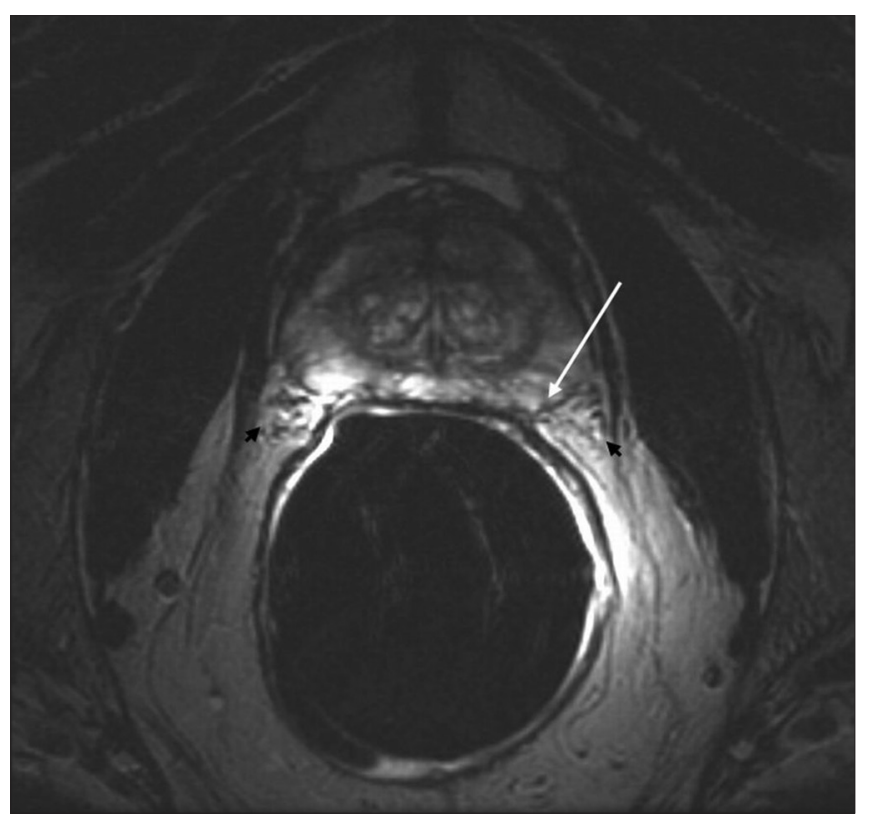

Figure 4: True capsule (arrow) and neurovascular bundles at 5 and 7 o'clock positions (black arrowheads) 
disease was highly likely to be present. Clinically significant disease was defined as a lesion with volume $\geq 0.5 \mathrm{~cm}^{3}$ and/ or Gleason score of $\geq 4+3 .^{[13]}$ The consensus on imaging, interpretation, scoring, and reporting is shown in Index 1.

The current standard of practice for performance of prostate MRI at 1.5-T uses the balloon endorectal surface coil (er-MRI) combined with pelvic phased-array coils, which yields high-resolution images with improved signal-to-noise ratio (SNR) and good spatial and spectral resolution. ${ }^{[14]}$ For MRI of prostate with endorectal coil, the patient is first placed in the left lateral decubitus position. After digital rectal examination, an endorectal balloon with a coil mounted inside is inserted into the rectum. The balloon is then inflated with $80 \mathrm{cc}$ of air, which helps to homogenize the magnetic field by reducing the susceptibility differences between the balloon and the prostate. The patient is then placed in the supine position with pelvic phased-array coils. The position of the endorectal coil is first checked on scout images and repositioned if necessary, as appropriate placement of the coil is essential for optimum image quality. At our institute, a standard protocol is followed [Index 2]. Spectroscopy is not routinely performed.

\section{Imaging Characteristics of Prostate Cancer on Conventional Magnetic Resonance Imaging}

\section{Organ-confined prostate cancer}

On T2W imaging, peripheral zone cancer foci are seen as rounded or ill-defined low-signal-intensity lesions [Figure 7]. This appearance is, however, mimicked by several other entities such as prostatitis, hemorrhage, benign hyperplasia, atrophy, and treatment-related changes.$^{[14]}$ It is advisable that the radiologist correlate the imaging findings with the timing and inference of prostate biopsy, serum prostate-specific antigen (PSA) level, the Gleason score, and the clinical findings on digital rectal examination. T1W images allow distinction between the post-biopsy hemorrhage [Figure 8], which appears hyperintense on T1W images, and the cancer foci. To allow precise diagnosis and avoid false negative interpretation because of overlying hemorrhage, prostate MRI is usually performed 6-8 weeks after the endorectal biopsy. ${ }^{[15]}$ The transitional zone tumors appear as low-signal-intensity lesions on T2W images and show a lenticular shape, with ill-defined margins and absence of an appreciable capsule ${ }^{[16]}$ [Figure 9]. Localization of tumor in the central gland is very difficult in the setting of stromal hyperplasia because of the background heterogeneity ${ }^{[6]}$ However, features such as well-defined margins, visible capsule, and round shape favor a diagnosis of benign prostatic hyperplasia (BPH) nodules. The anterior fibro muscular stroma can be invaded by transitional zone tumors. BPH nodules, on the other hand, may displace but do not invade the fibromuscular stroma ${ }^{[16]}$ [Figure 10].

\section{Extracapsular extension}

The diagnosis of ECE upstages the tumor to $\mathrm{T}_{3}$, thereby changing the management plan and prognosis. Therefore,
ECE is best detected by high-resolution multiplanar T2W images. The MRI features suggestive of ECE - and the misleading signs - are described in Table $1 . \cdot^{[9,17-21]}$

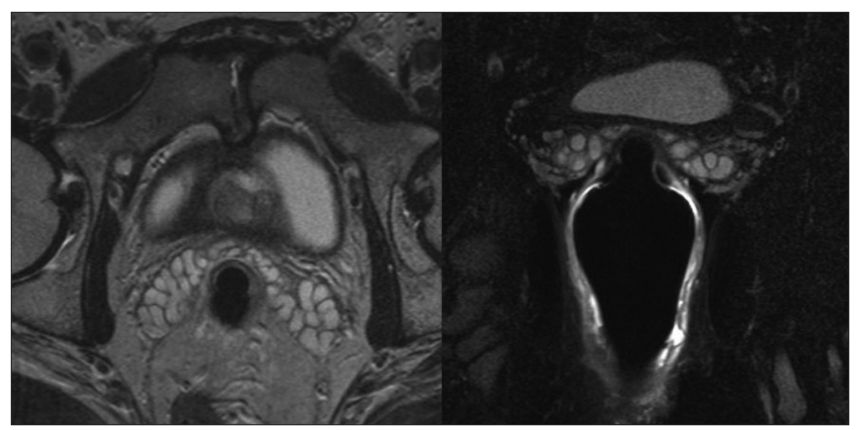

Figure 5: T2-weighted axial and coronal images show normal appearance of seminal vesicles

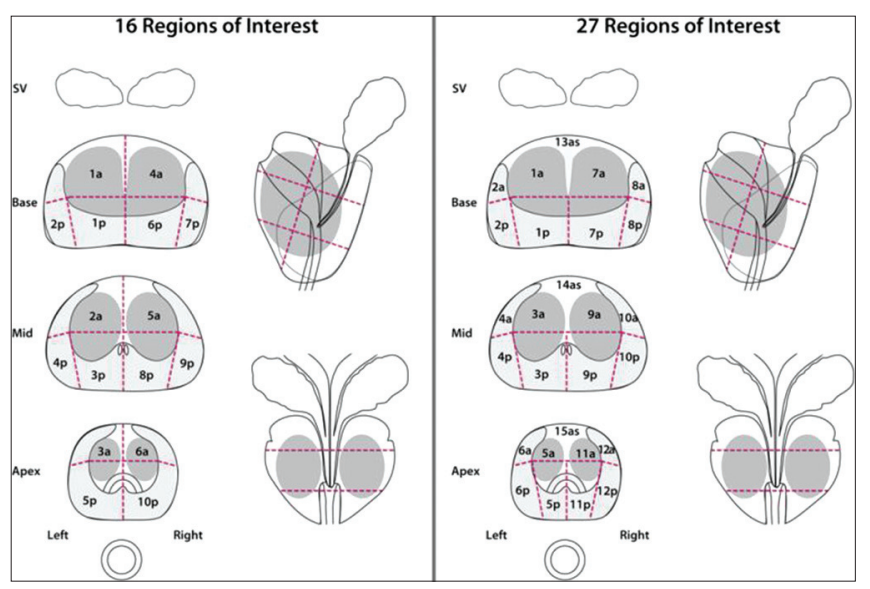

Figure 6: The European Consensus Guidelines division of the prostate gland into the minimal 16 - and optimal 27 - regions of interest

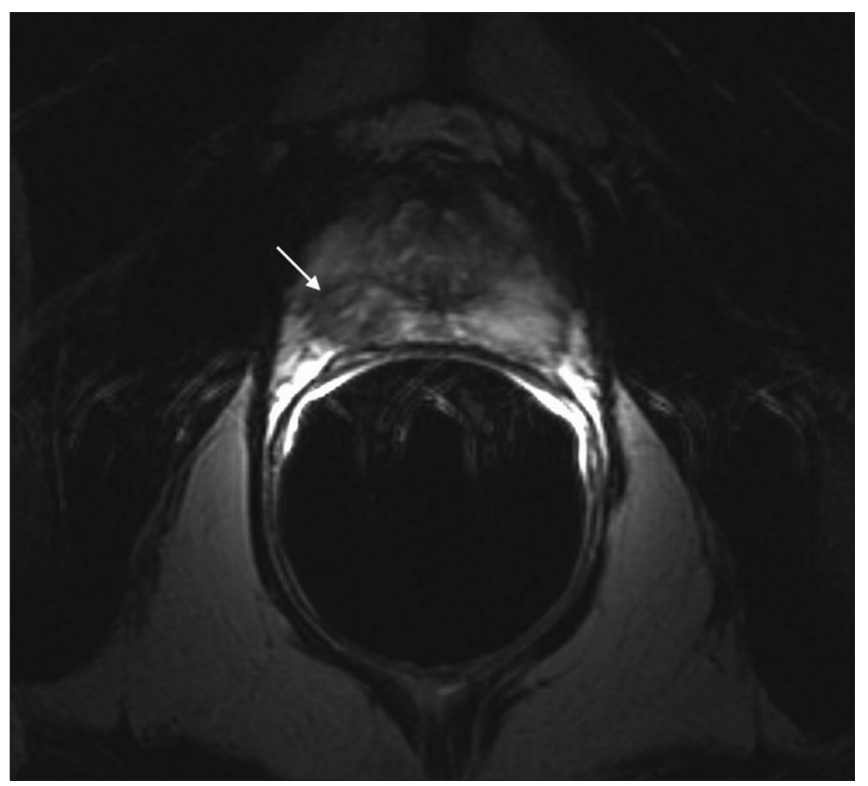

Figure 7: T2-weighted axial images show hypointense tumor focus (arrow) in the left basal peripheral zone. Organ-confined cancer was confirmed on surgical histopathology 
Table 1: Extracapsular extension and misleading signs

\section{MRI features of ECE [Figure 11A and B]}

Visualization of extension of hypointense tumor in hyperintense periprostatic adipose tissue or adjacent structures like rectum/bladder

Secondary features

Asymmetric neurovascular bundles

Encasement of neurovascular bundles by the tumor

Irregular margin of the gland

Capsular obscuration

Broad tumor contact with the capsular surface

Obliteration of retroprostatic angle

Seminal vesicle invasion is seen as extension of hypointense tumor into hyperintense seminal vesicles

Misleading signs

Irregular bulging in nonpalpable tumors

Thickened walls and asymmetric widening of seminal vesicles (can be seen in benign conditions like senile amyloidosis)

ECE: Extracapsular extension

Table 2: Anatomic stage/diagnostic groups

\begin{tabular}{|c|c|c|c|c|c|}
\hline Group & $T$ & $\mathrm{~N}$ & $\mathrm{M}$ & PSA & Gleason \\
\hline \multirow[t]{3}{*}{ I } & $T_{1 a-c}$ & $\mathrm{~N}_{0}$ & $\mathrm{M}_{0}$ & $<10$ & $\leq 6$ \\
\hline & $\mathrm{T}_{2 \mathrm{a}}$ & $\mathrm{N}_{0}$ & $\mathrm{M}_{0}$ & $<10$ & $\leq 6$ \\
\hline & $T_{1-2 a}$ & $\mathrm{~N}_{0}$ & $M_{0}$ & $x$ & $x$ \\
\hline \multirow[t]{6}{*}{$\| A$} & $\mathrm{~T}_{1 \mathrm{a}-\mathrm{c}}$ & $\mathrm{N}_{0}$ & $\mathrm{M}_{0}$ & $<20$ & 7 \\
\hline & $T_{1 a-c}$ & $\mathrm{~N}_{0}$ & $\mathrm{M}_{0}$ & $\geq 10<20$ & $\leq 6$ \\
\hline & $\mathrm{T}_{2 \mathrm{a}}$ & $\mathrm{N}_{0}$ & $\mathrm{M}_{0}$ & $\geq 10<20$ & $\leq 6$ \\
\hline & $\mathrm{T}_{2 \mathrm{a}}$ & $\mathrm{N}_{0}$ & $\mathrm{M}_{0}$ & $<20$ & 7 \\
\hline & $T_{2 b}$ & $\mathrm{~N}_{0}$ & $M_{0}$ & $<20$ & $\leq 7$ \\
\hline & $\mathrm{T}_{2 \mathrm{~b}}$ & $\mathrm{~N}_{0}$ & $\mathrm{M}_{0}$ & $x$ & $x$ \\
\hline IIB & $\mathrm{T}_{2 \mathrm{c}}$ & $\mathrm{N}_{0}$ & $M_{0}$ & Any & Any \\
\hline \multirow[t]{3}{*}{ III } & $T_{1-2}$ & $\mathrm{~N}_{0}$ & $M_{0}$ & $\geq 20$ & Any \\
\hline & $T_{1-2}$ & $\mathrm{~N}_{0}$ & $\mathrm{M}_{0}$ & Any & $\geq 8$ \\
\hline & $T_{1 a-b}$ & $\mathrm{~N}_{0}$ & $\mathrm{M}_{0}$ & Any & Any \\
\hline \multirow[t]{3}{*}{ IV } & $\mathrm{T}_{4}$ & $\mathrm{~N}_{0}$ & $M_{0}$ & Any & Any \\
\hline & Any $\mathrm{T}$ & $\mathrm{N}_{1}$ & $M_{0}$ & Any & Any \\
\hline & Any $T$ & Any N & $\mathrm{M}_{1}$ & Any & Any \\
\hline
\end{tabular}

PSA: Prostate-specific antigen, T: Primary tumor, N: Regional lymph nodes, M: Distant metastasis

\section{Staging}

Staging of the prostate cancer is based on local, nodal, and distant extent of disease and is essential for risk stratification and assessment of prognosis. The salient points from the American Joint Committee on Cancer staging manual ( $7^{\text {th }}$ edition; 2010) are summarized below. The diagnostic groups based on staging and PSA and Gleason score are mentioned in Table 2.

\section{Primary tumor}

\section{Clinical:}

- $\mathrm{T}_{\mathrm{x}}$ : Primary tumor cannot be assessed

- $\mathrm{T}_{0}$ : No evidence of primary tumor

- $\mathrm{T}_{1}$ : Clinically inapparent tumor, neither palpable nor visible by imaging

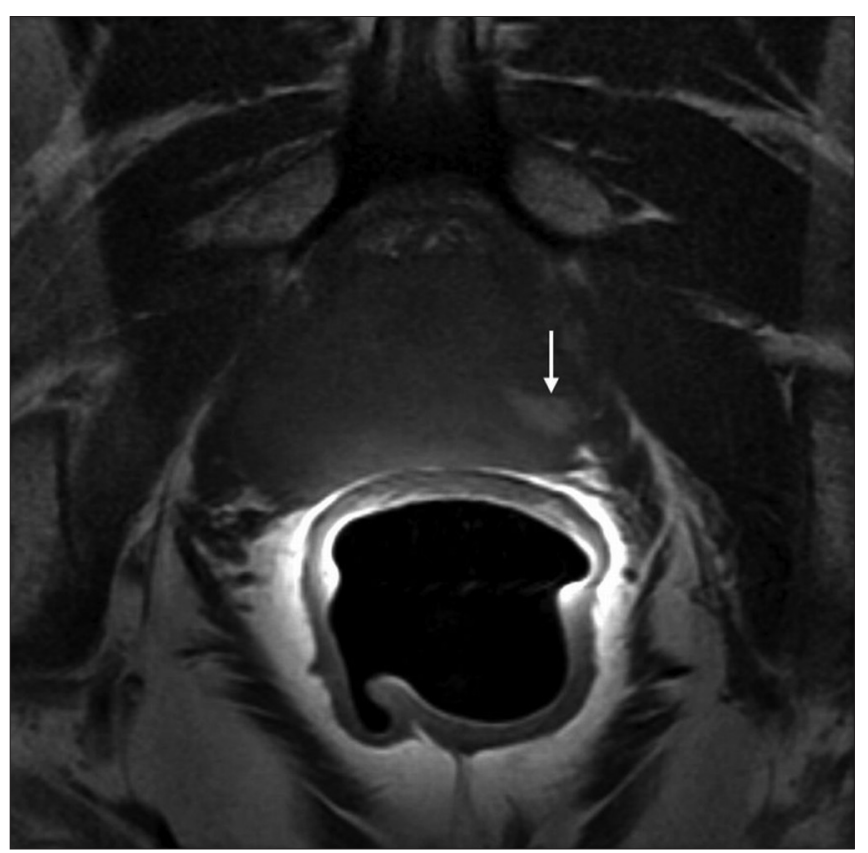

Figure 8: Postbiopsy hemorrhage. T1-weighted images show hyperintense focus (arrow) in the left peripheral zone, consistent with postbiopsy hemorrhage

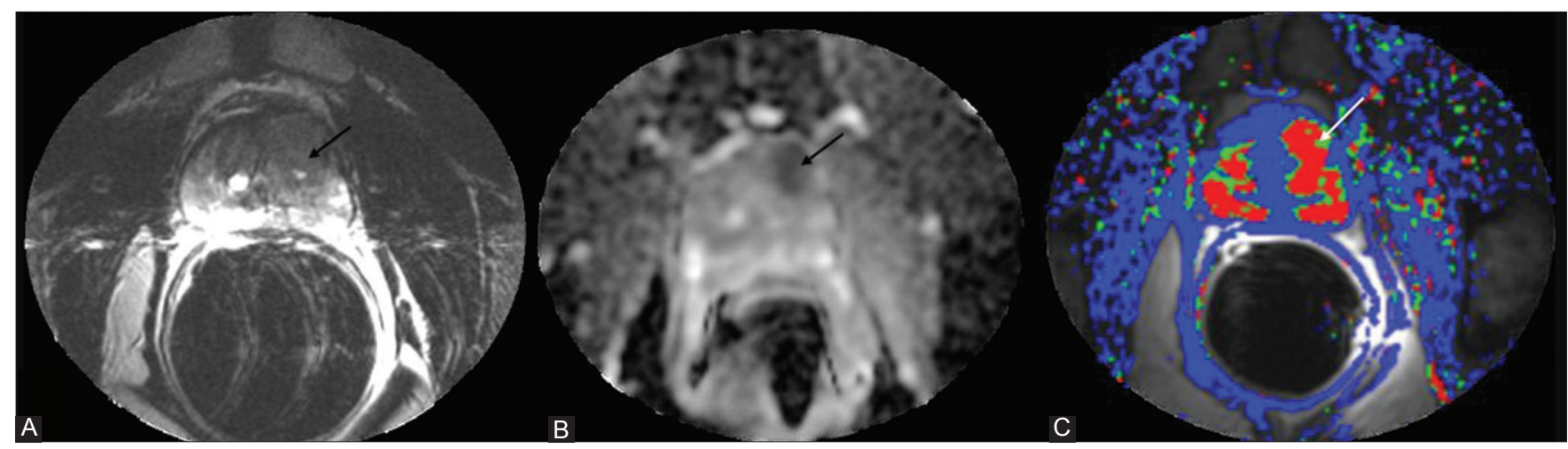

Figure 9: Organ-confined transitional zone tumor. (A) Axial T2-weighted image shows dark signal in the left tumor focus; (B) the tumor shows restricted diffusion and low ADC (arrow); $(C)$ dynamic contrast-enhanced magnetic resonance imaging with quantitative color-coded map of reverse flow of contrast medium (Kep) shows elevated values within the tumor focus (arrow) 
- $\mathrm{T}_{1 \mathrm{a}}$ : Tumor incidental; histologic finding in $\leq 5 \%$ of tissue resected

- $\mathrm{T}_{1 \mathrm{~b}}$ : Tumor incidental; histologic finding in $>5 \%$ of tissue resected

- $\mathrm{T}_{1 \mathrm{c}}$ : Tumor identified by needle biopsy (e.g., because of elevated PSA)

- $\mathrm{T}_{2}$ : Tumor confined within prostate

- $\mathrm{T}_{2 \mathrm{a}}$ : Tumor involves one-half of one lobe or less

- $\mathrm{T}_{2 \mathrm{~b}}$ : Tumor involves more than one-half of one lobe but not both lobes

- $\mathrm{T}_{2 \mathrm{c}}$ : Tumor involves both lobes

- $\mathrm{T}_{3}$ : Tumor extends through the prostate capsule

- $\mathrm{T}_{3 \mathrm{a}}: \mathrm{ECE}$ (unilateral or bilateral)

- $\mathrm{T}_{3 \mathrm{~b}}$ : Tumor invades seminal vesicle(s)

- $\mathrm{T}_{4}$ : Tumor is fixed or invades adjacent structures other than seminal vesicles, e.g., external sphincter, rectum, bladder, levator muscles, and/or pelvic wall [Figure 12].

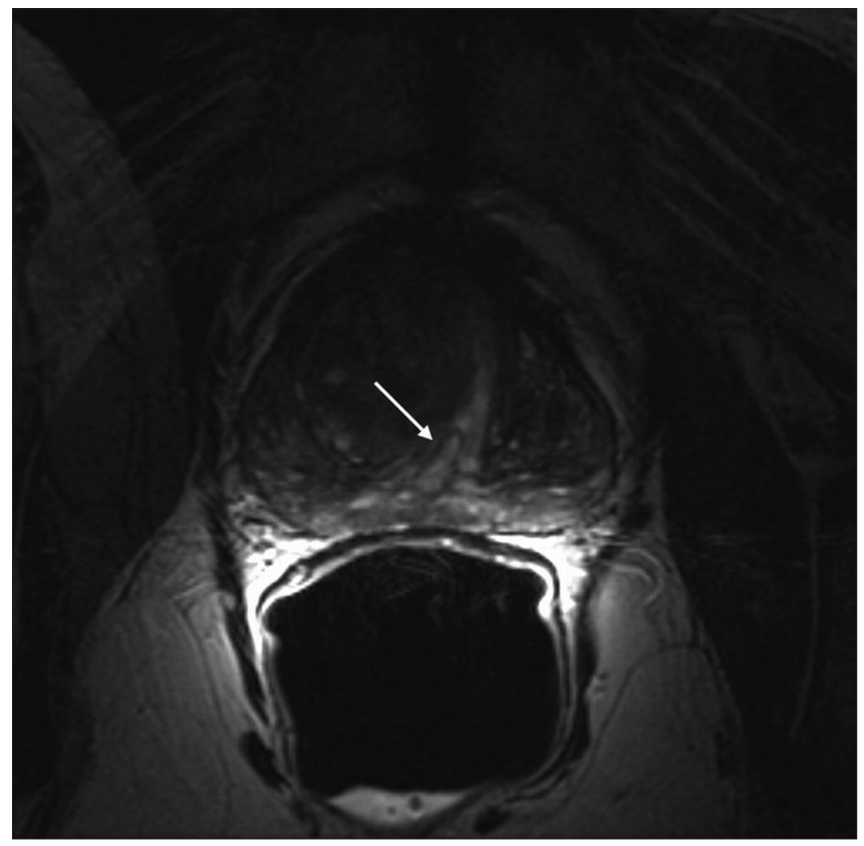

Figure 10: T2-weighted image shows benign prostatic hyperplasia nodule in the transitional zone. Note the displacement of the fibromuscular stroma (arrow)

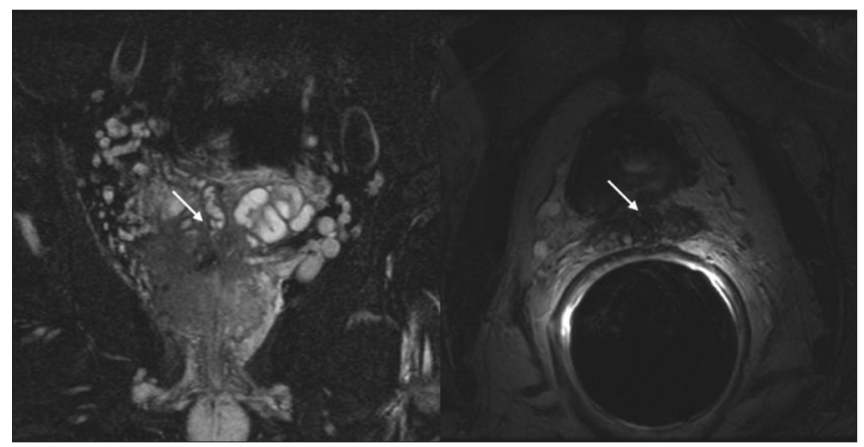

Figure 11 (B): Signs of ECE-seminal vesicle involvement noted on coronal and axial images

\section{Regional lymph nodes}

Clinical:

- $\mathrm{N}_{\mathrm{x}}$ : Regional lymph nodes (N) not assessed

- $\mathrm{N}_{0}$ : No regional lymph node metastasis

- $\mathrm{N}_{1}$ : Metastasis in regional lymph node(s).

\section{Distant metastasis}

- $\mathrm{M}_{0}$ : No distant metastasis(M)

- $\mathrm{M}_{1}$ : Distant metastasis

- $\mathrm{M}_{1 \mathrm{a}}$ : Nonregional lymph node(s)

- $\mathrm{M}_{1 \mathrm{~b}}$ : Bone(s) [Figure 13]

- $\mathrm{M}_{1 \mathrm{c}}$ : Other site(s), with or without bone disease.

Detection of metastatic disease

The likelihood of detecting metastasis is higher when the tumor is $\mathrm{T}_{2}$ or higher, serum PSA level is $>20 \mathrm{ng} / \mathrm{ml}$, and Gleason score is $\geq 7$. ${ }^{[22]}$ Prostate cancer can metastasize by either the lymphatic or the hematogenous route. The lymph nodal groups that are often involved are obturator, internal iliac, common iliac, and presacral [Figure 14]. The site of the involved lymph node is an important parameter as it affects the staging; pelvic nodal disease is considered as $\mathrm{N}_{1}$, and common iliac or retroperitoneal nodal disease is considered as $M_{1}$. Disease progression and survival is affected by the number of lymph nodes involved. If more than five lymph nodes are involved, recurrence-free 10-year survival is

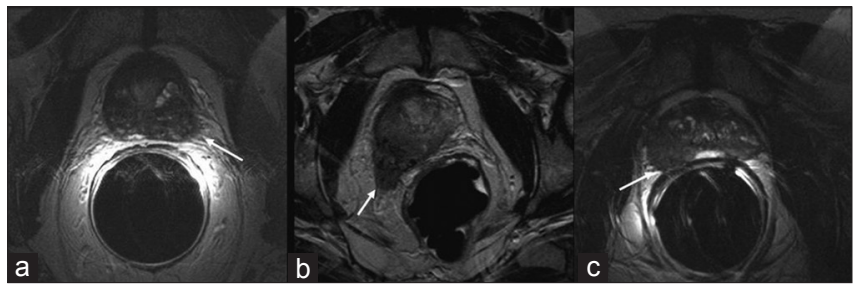

Figure 11 (A): Signs of extracapsular extension (ECE) on T2-weighted magnetic resonance imaging: (a) Irregular margin of the peripheral zone (arrow); (b) involvement of neurovascular bundles (arrow); (c) obliteration of rectoprostatic angle (arrow)

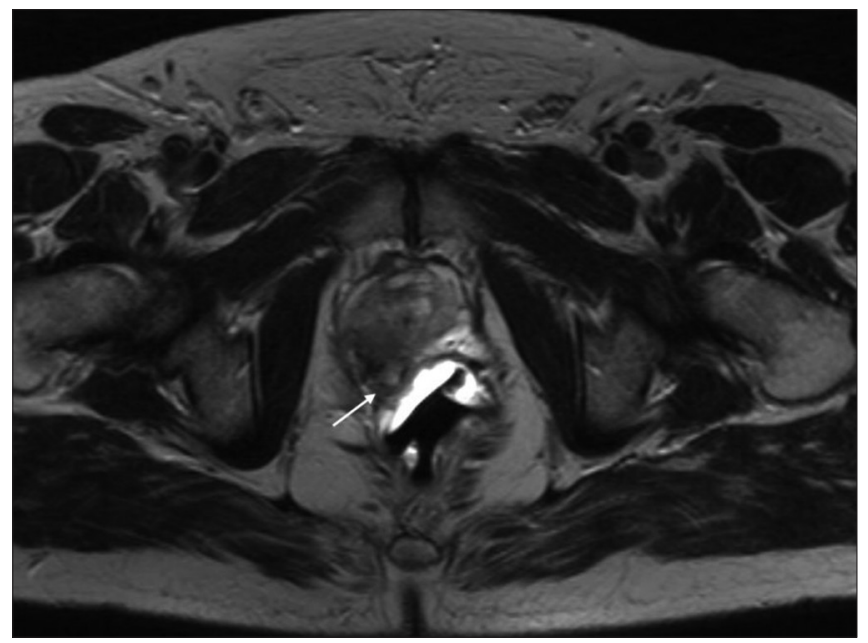

Figure 12: $T_{4}$ stage disease. Axial T2-weighted image shows involvement of rectal wall (arrow) 
$49 \%$, as compared with $70 \%$ in patients with $1-2$ lymph nodes ${ }^{[23]}$ Hematogenous metastasis is seen most commonly in lumbar vertebrae, pelvic bones, ribs, and proximal ends of the femora. Visceral metastases are rare compared with bony metastases. ${ }^{[22,24]}$

To improve the diagnostic accuracy, advanced MRI techniques like diffusion-weighted imaging (DWI), dynamic contrast-enhanced MRI (DCE-MRI), MR spectroscopy, and lymphotrophic superparamagnetic nanoparticle-enhanced MRI (LSN-MRI) are being increasingly used.

\section{Diffusion-Weighted Imaging}

DWI exploits the property of constant Brownian motion of the water molecules in tissue. ${ }^{[25]}$ This property is affected by increased cellularity, tissue organization, extracellular space, and integrity of cell membranes. ${ }^{[2]}$ Prostate cancer foci are composed of tightly packed cellular elements with reduced extracellular space, which can be visualized on DWI images as areas of restricted diffusion (high signal intensity), with corresponding low signal intensity on Apparent Diffusion Coefficient (ADC) maps [Figure 15]. ${ }^{[27]}$ With the advent of parallel imaging, high field-strength magnets, and endorectal coils, it is now possible to obtain DWI with improved SNR. At our institution, DWI is a part of our routine prostate cancer imaging protocol. Diffusion in normal prostatic tissue is variable. The ADC map shows significantly higher signal (less restricted diffusion) in the peripheral zone due to the high proportion of glandular tissue. ${ }^{[28]}$ The ADC and DWI are affected by age as well. ${ }^{[29]}$ Haider et al. demonstrated that the combination of a combined T2W images and DWI performed better than the former alone in the detection of peripheral zone tumors that were $>4 \mathrm{~mm}$ and had Gleason score of $\geq 6 .{ }^{[30]}$ High lesion conspicuity on DWI is especially important for guiding second biopsy in patients with high clinical suspicion for tumor but negative first biopsy. ${ }^{[31]}$ Furthermore, Kim et al. demonstrated that DWI combined with T2W images have better diagnostic performance than the latter alone in predicting invasion of seminal vesicles. ${ }^{[32]}$ DWI, however, only distinguishes cancer foci if the tumor/volume ratio is $50 \%$ or more. ${ }^{[33]}$ DWI can also be used for detection of recurrent tumor as shown in a recent study by Kim et al. The sensitivity and specificity reported by them were $25 \%$ and $57 \%$, respectively, for $\mathrm{T} 2 \mathrm{~W}$ images alone compared to $62 \%$ and $91 \%$, respectively, for combined T2WI and DWI. The mean ADC noted of recurrent tumor was $0.98 \times 10^{-3} \mathrm{~mm}^{2} / \mathrm{s}$ and that of normal tissue was $1.60 \times 10^{-3} \mathrm{~mm}^{2} / \mathrm{s} \cdot{ }^{[34]}$ Despite its advantages, DWI has drawbacks such as nonstandardized protocols, image distortion, susceptibility artifacts, and decreased specificity because of considerable overlap between malignant and benign conditions; for example, $\mathrm{BPH}$ and prostatitis have altered cellular density and interstitial pressure and thereby can mimic cancer on DWI. ${ }^{[26,35]}$
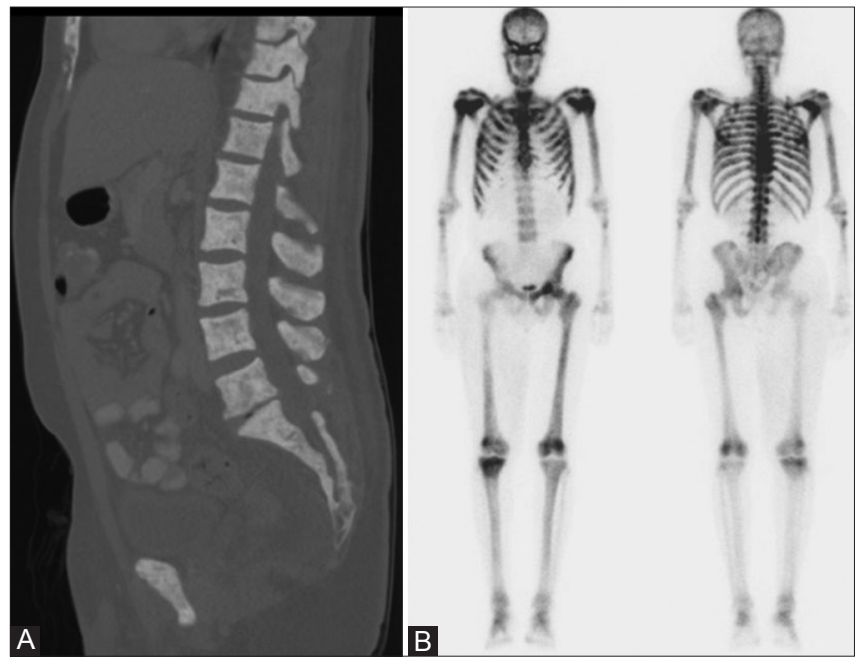

Figure 13: Bone metastasis. (A) Sagittal-reformatted computed tomography images show diffuse sclerotic spinal metastatic lesions in a patient with prostate cancer; (B) corresponding bone superscan

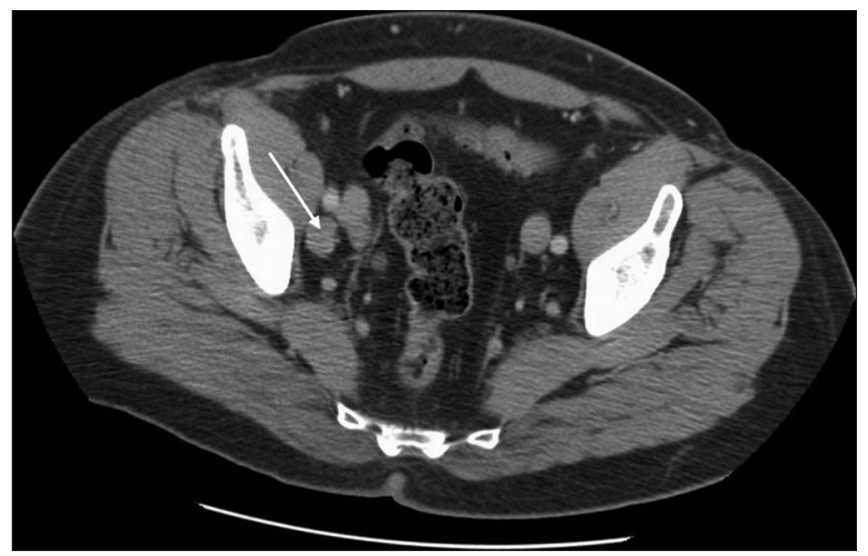

Figure 14: Axial contrast-enhanced computed tomography image shows a right obturator lymph node metastasis in a patient with prostate carcinoma

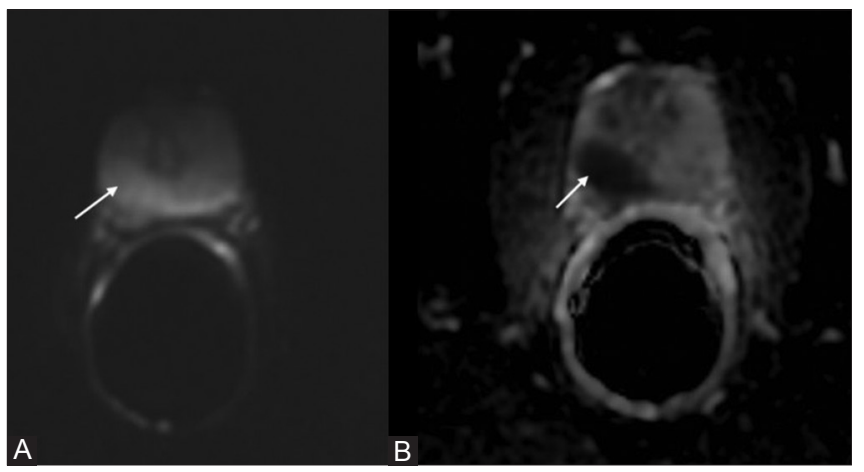

Figure 15: (A) Diffusion-weighted imaging $(b=800)$ shows hyperintense tumor focus in the right peripheral zone, with the tumor appearing dark on the corresponding ADC image (B)

\section{Dynamic Contrast-Enhanced Magnetic Resonance Imaging}

The basic principle of DCE-MRI is related to tumor angiogenesis. Any tumor $>2 \mathrm{~mm}$ inevitably shows 
angiogenesis. ${ }^{[36]}$ Prostate cancer cells by expressing (VEGF) vascular endothelial growth factor are no exception. ${ }^{[37]}$ There is discrepancy between the interstitial space of cancer tissue and normal tissue. ${ }^{[3]}$ Due to large interstitial spaces, there is difference in the concentration of intravenous contrast material between intravascular and extravascular spaces, which accentuates contrast transfer through vascular walls and thus results in the unique enhancement pattern of strong early enhancement and rapid washout. ${ }^{[38,39]}$ DCE-MRI provides quantitative parameters reflecting the permeability and flow characteristics of vessels within the lesion. ${ }^{[14]}$ The enhancement curves generated from DCE-MRI are fit to a pharmacokinetic model like Toft's. This generates the following quantitative parameters:

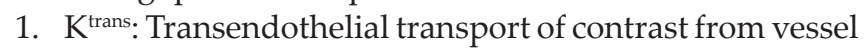
lumen to tumor interstitium, i.e., permeability

2. Ve: Fractional volume of extravascular extracellular space

3. Kep: Reverse transport parameter of contrast medium back to the vascular space.

In prostatic cancer foci, these values are significantly higher than that of normal tissue [Figure 16]. ${ }^{[31,40,41]}$

At our institute, for DCE-MRI, 20 cc gadolinium is injected at a rate of $3 \mathrm{cc}$ per second and serial T1W 3D images are obtained every $2-5 \mathrm{~s}$ through the entire prostate.

A recent study by Jackson et al. showed that the sensitivity and specificity of DCE-MRI (50\% and 85\%, respectively) is higher than that of $\mathrm{T} 2 \mathrm{~W}$ imaging $(21 \%$ and $81 \%$, respectively) ${ }^{[42]}$ Because of the increased microvessel density, DCE can help distinguish carcinomatous foci from BPH nodules. ${ }^{[43]}$ Yoshizako et al. demonstrated that DCE-MRI can be used as a complementary tool along with DWI and T2W images, with the combination yielding a specificity of $93.8 \%$ and positive predictive value $94.7 \%{ }^{[44]}$ For the detection of recurrent prostatic carcinomas after electron beam radiation therapy (EBRT), DCE-MRI has a better sensitivity and positive and negative predictive values $(72 \%, 46 \%$, and $95 \%$, respectively) than $\mathrm{T} 2 \mathrm{~W}$ imaging alone. ${ }^{[45]}$ In patients post radical prostatectomy, the sensitivity and specificity has been shown to be $84.1 \%$ and $89.3 \%$, respectively. ${ }^{[46]}$

\section{MR Spectroscopy}

MR spectroscopy provides information about the biochemical and metabolic status of the tissue. MR spectroscopy evaluates the gland in three dimensions with voxel size of $0.24-0.34 \mathrm{~cm}^{3}$ using chemical shift imaging (CSI) and point-resolved spectroscopy (PRESS). ${ }^{[47]}$ The metabolic data is superimposed on the MR images to identify and localize the cancer. ${ }^{[31]}$ The normal prostatic tissue is citrate rich, with low choline and creatine levels in the peripheral zone. In the central and transitional zones, the citrate levels are lower than in the peripheral zones. ${ }^{[4]}$ The fibromuscular stroma and periurethral tissues have even lower levels of citrate. Instead of the normal high citrate metabolism, cancer cells utilize citrate oxidizing metabolism. ${ }^{[38,49]}$ High turnover of phospholipids raises the choline level and thus increases the choline/citrate ratio. This can be used to detect malignancy in the peripheral zone. Creatine, on the other hand, is a marker of cellular energy storage and the levels are not significantly different between healthy and cancerous prostatic tissue. The creatine peak may be indistinguishable from the choline peak because of the close proximity between the two. The mean normal choline + creatine/citrate ratio is $0.22 \pm 0.0013$ at $1.5-\mathrm{T} .{ }^{[50]}$ Peripheral zone voxels with choline and creatine ratio to citrate that are $>3 \mathrm{SD}$ (standard deviations) above average is considered as highly suggestive of cancer [Figure 17]. ${ }^{[50]}$ The exact ratio is affected by factors such as magnet strength and settings. The 5-point scale devised by Jung et al. is reasonably accurate, with excellent interobserver agreement, in distinguishing benign from malignant tissue. According to this scale: 1 = probably benign; 2 = possibly benign; 3 = equivocal; 4 = possibly malignant; and 5 = probably malignant. ${ }^{[50]}$ The spectra can be affected by postbiopsy hemorrhage, which can degrade it, and also by prostatitis and $\mathrm{BPH}$, which can mimic carcinoma. ${ }^{[1,51,52]}$ Coupled with MRI, MR spectroscopy is shown to have sensitivity and

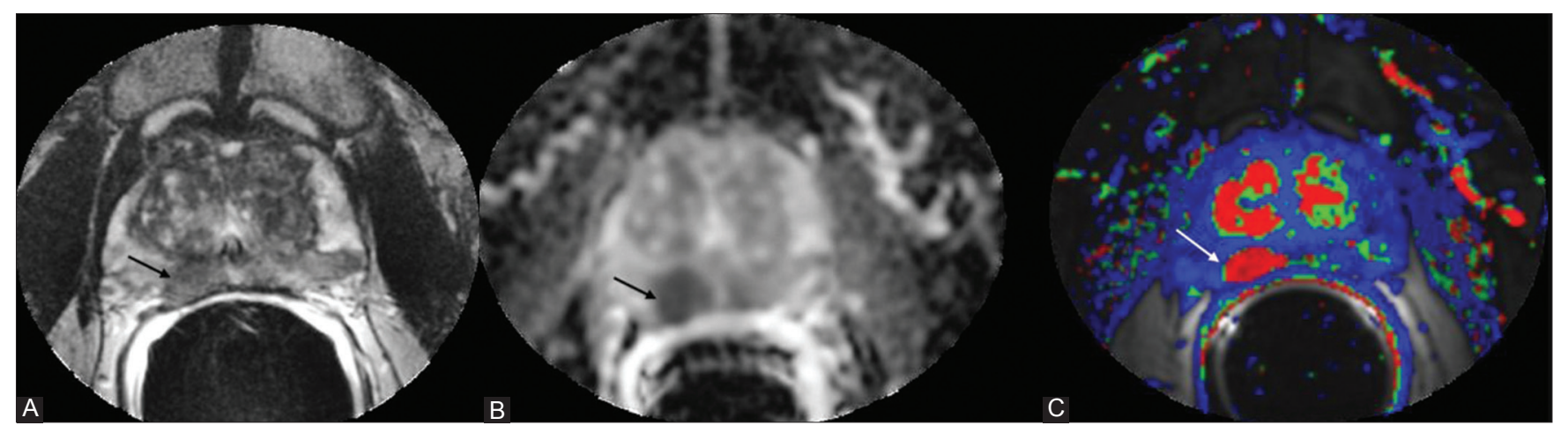

Figure 16: Organ-confined right peripheral zone tumor. (A) Axial T2-weighted image shows dark signal in the right peripheral zone. (B) The tumor shows restricted diffusion and low ADC (arrow); (C) dynamic contrast-enhanced magnetic resonance imaging with quantitative color-coded map of reverse flow of contrast medium (Kep) shows elevated values within the tumor focus (arrow) 


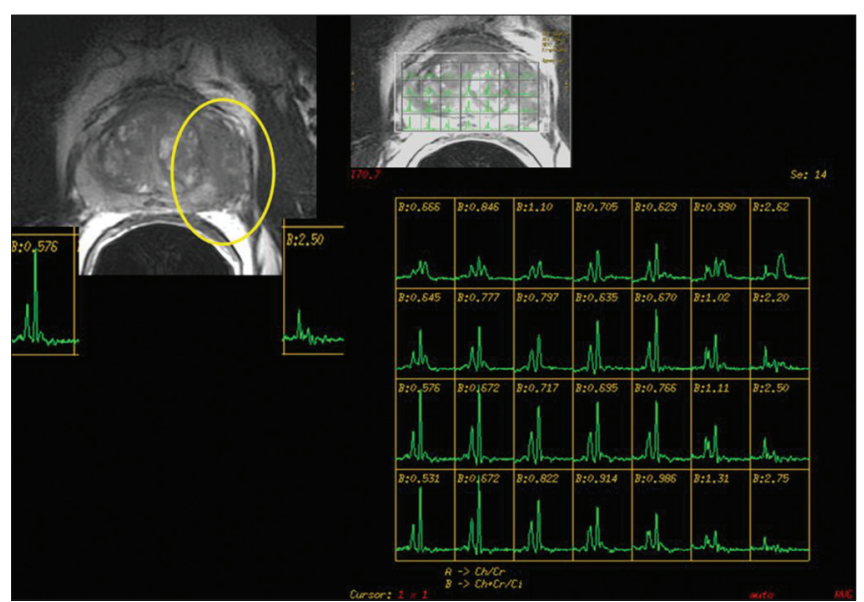

Figure 17: A 48-year-old male with prostate-specific antigen of $5.43 \mathrm{ng} / \mathrm{ml}$. Axial T2 fast spin echo (FSE) image demonstrates a hypointense mass occupying the peripheral zone of the left mid-gland and an irregular prostate capsule. Magnetic Resonance Spectroscopy Imaging (MRSI): The left mid-gland mass spectra demonstrates a high choline-to-citrate ratio in multiple voxels, indicative of high-volume disease and extracapsular extension. Prostatectomy revealed left mid-gland Gleason grade $9(5+4)$ adenocarcinoma, stage $T_{3 a}$

specificity of $95 \%$ and $91 \%$, respectively, for intraprostatic tumor localization. ${ }^{[53]}$ MR spectroscopy can also depict metabolic atrophy post treatment, which can be used for distinguishing post-treatment changes from recurrence. ${ }^{[54]}$

\section{Lymphotrophic Superparamagnetic Nanoparticle-Enhanced Magnetic Resonance Imaging}

Lymphotrophic superparamagnetic nanoparticle-enhanced magnetic resonance imaging (LSN-MRI) was developed recently and has been validated by multiple clinical trials. Reticuloendothelial cells in lymph nodes show uptake of these nanoparticles typically 48 hours post intravenous injection [Figure 18]. In nodal spread of malignancies, the reticuloendothelial cells are replaced by tumor cells, which fail to show normal uptake of iron thereby increasing specificity of nodal involvement by tumor irrespective of size criterion which is known for false negative results.

For characterizing lymph nodes in prostatic cancer the highest sensitivity reported was by Harisinghani et al., who reported sensitivity of $100 \%$ (with specificity of $96 \%$ ) with metastatic nodes outside the classical field of lymph node dissection in $11 \% .{ }^{[5]}$ In another study by Heesakkers et al., the positive predictive value was $69 \%$ and negative predictive value was $96 \%{ }^{[56]} \mathrm{LSN}-\mathrm{MRI}$ is thus a noninvasive functional imaging tool that has potential for improving preoperative staging.

\section{Suimmary}

There have been remarkable technical advances in multiparametric MRI and its role in detecting, localizing,

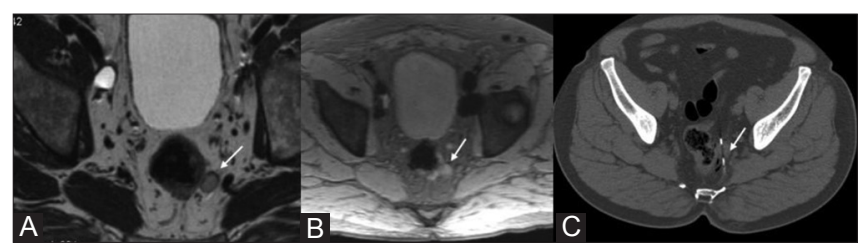

Figure 18: Lymphotrophic superparamagnetic nanoparticle-enhanced magnetic resonance imaging. (A) Axial T2-weighted image shows a left perirectal node. (B) Axial post-ultrasmall super paramagnetic iron oxide (USPIO) T2* -gradient-echo image shows hyperintense node (arrow), indicating lack of USPIO uptake within the node, (C) Metastasis was proven on computed tomography -guided lymph node biopsy

and staging prostate cancer, as well as in evaluating local recurrence after treatment. However, consistency in conducting er-MRI and its interpretation is the key to the widespread use of this multiparametric imaging tool. A multipronged approach and the combined use of these techniques can improve diagnostic performance, provided the radiologist understands the advantages and limitations of each technique.

\section{Acknowledgement}

Authors of this manuscript would like to acknowledge Dr Sadhna Verma, MD for providing the valuable image input on MR Spectroscopy.

\section{Index 1}

Areas of consensus on imaging interpretation, scoring, and reporting: ${ }^{[13]}$

1. Areas of positive consensus

- When scoring the prostate for the presence or absence of cancer for T2W, diffusion-weighted, contrast-enhanced, and MR spectroscopy sequences, the range of scores should be 1-5 for each imaging type.

- Both individual lesions and areas of the prostate should be separately scored for probability of malignancy.

- The maximum diameter of the largest abnormal lesion should be recorded.

- The following should be scored for involvement, with an individual scoring range of 1-5:

- ECE

- Seminal vesicles (extra-and intraprostatic)

- Distal sphincter

- Rectal wall

- Neurovascular bundles

- Bladder neck.

- As a minimum requirement, the prostate should be divided into 16 regions of interest (apical, mid, and base quadrants) and, as an optimum requirement, into 27 regions of interest.

- The ADC value should be stated for any suspicious lesion detected. 
- Dynamic contrast-enhanced should be scored according to the morphological enhancement pattern.

- The following clinical information is important for reporting the imaging and should be included:

- PSA level

- Digital rectal examination findings

- Time scale since prostate biopsies, and results of previous biopsies

- Results of previous MRI scans

- History of previous prostate treatment or intervention (e.g., transurethral resection of prostate, prostate radiotherapy)

- History of medical treatment (e.g., 5 - $\alpha$-reductase inhibitors, hormones).

- As a minimum requirement, each MRI should be assessed and scored by one radiologist and, as an optimal requirement, scored by two radiologists independently, with discrepancies referred for consensus.

- If one of the modalities within the minimum dataset is noninterpretable due to artefact, the denominator of the scoring system should be changed to allow for the lack of score for the affected sequence.

- Dedicated software for imaging interpretation should be developed for this purpose, with the ability to display, co-register, segment, fuse, and analyze every tool in an integrated single workspace.

- The final report should be presented electronically, in both number and picture form, and should include relevant images.

2. Areas lacking consensus

- Areas of the prostate should be scored separately rather than by individual lesions.

- The overall score for probability of tumor given by the radiologist should be influenced by other clinical results (e.g., PSA level).

- The overall score should be based purely on imaging appearances.

- A separate radiologist's "hunch" score should be given that represents the radiologist's personal hunch on the likelihood of malignancy, regardless of the objective radiologic score.

- The final score should be given as individual scores, a sum of the individual scores, or as a radiologist's overall opinion score.

- T-staging should be a formal part of the final report.

\section{Index 2}

1. Protocol: 1.5- or 3.0-T MRI technique

- T1W axial sequence

- T2W axial sequence

- $\mathrm{T} 2 \mathrm{~W}$ coronal sequence

- T2W sagittal sequence

2. DWI and ADC maps

3. Postcontrast DCE sequences

\section{References}

1. SEER stat fact sheets: Prostate [Internet]. Available from: http://seer. cancer.gov/statfacts/html/prost.html\#prevalence. [Last accessed on 2011 Apr 7].

2. Trabulsi EJ, Sackett D, Gomella LG, Halpern EJ. Enhanced transrectal ultrasound modalities in the diagnosis of prostate cancer. Urology 2010;76:1025-33.

3. Jemal A, Center MM, DeSantis C, Ward EM. Global patterns of cancer incidence and mortality rates and trends. Cancer Epidemiol Biomarkers Prev 2010;19:1893-907.

4. Fütterer JJ, Barentsz JO, Heijmink SW. Value of 3-T magnetic resonance imaging in local staging of prostate cancer. Top Magn Reson Imaging 2008;19:285-9.

5. McNeal JE, Redwine EA, Freiha FS, Stamey TA. Zonal distribution of prostatic adenocarcinoma. Correlation with histologic pattern and direction of spread. Am J Surg Pathol 1988;12:897-906.

6. Ravizzini G, Turkbey B, Kurdziel K, Choyke PL. New horizons in prostate cancer imaging. Eur J Radiol 2009;70:212-26.

7. Verma S, Rajesh A. A clinically relevant approach to imaging prostate cancer: Review. AJR Am J Roentgenol 2011;196:S1-10.

8. Cruz M, Tsuda K, Narumi Y, Kuroiwa Y, Nose T, Kojima Y, et al. Characterization of low-intensity lesions in the peripheral zone of prostate on pre-biopsy endorectal coil MR imaging. Eur Radiol 2002;12:357-65.

9. Claus FG, Hricak H, Hattery RR. Pretreatment evaluation of prostate cancer: Role of MR imaging and $1 \mathrm{H}$ MR spectroscopy. Radiographics 2004;24:S167-80.

10. Allen KS, Kressel HY, Arger PH, Pollack HM. Age-related changes of the prostate: Evaluation by MR imaging. AJR Am J Roentgenol 1989;152:77-81.

11. Puech P, Huglo D, Petyt G, Lemaitre L, Villers A. Imaging of organ-confined prostate cancer: Functional ultrasound, MRI and PET/computed tomography. Curr Opin Urol 2009;19:168-76.

12. Kim B, Kawashima A, Ryu JA, Takahashi N, Hartman RP, King BF Jr. Imaging of the seminal vesicle and vas deferens. Radiographics 2009;29:1105-21.

13. Dickinson L, Ahmed HU, Allen C, Barentsz JO, Carey B, Futterer JJ, et al. Magnetic resonance imaging for the detection, localisation, and characterisation of prostate cancer: Recommendations from a European consensus meeting. Eur Urol 2011;59:477-94.

14. Bouchelouche K, Turkbey B, Choyke P, Capala J. Imaging prostate cancer: An update on positron emission tomography and magnetic resonance imaging. Curr Urol Rep 2010;11:180-90.

15. Qayyum A, Coakley FV, Lu Y, Olpin JD, Wu L, Yeh BM, et al. Organ-confined prostate cancer: Effect of prior transrectal biopsy on endorectal MRI and MR spectroscopic imaging. AJR Am J Roentgenol 2004;183:1079-83.

16. Akin O, Sala E, Moskowitz CS, Kuroiwa K, Ishill NM, Pucar D, et al. Transition zone prostate cancers: Features, detection, localization, and staging at endorectal MR imaging. Radiology 2006;239:784-92.

17. Wang L, Mullerad M, Chen HN, Eberhardt SC, Kattan MW, Scardino PT, et al. Prostate cancer: Incremental value of endorectal MR imaging findings for prediction of extracapsular extension. Radiology 2004;232:133-9.

18. Sala E, Akin O, Moskowitz CS, Eisenberg HF, Kuroiwa K, Ishill NM, et al. Endorectal MR imaging in the evaluation of seminal vesicle invasion: Diagnostic accuracy and multivariate feature analysis. Radiology 2006;238:929-37.

19. Yu KK, Hricak H, Alagappan R, Chernoff DM, Bacchetti P, Zaloudek CJ. Detection of extracapsular extension of prostate carcinoma with endorectal and phased-array coil MR imaging: Multivariate feature analysis. Radiology 1997;202:697-702. 
20. Fütterer JJ, Barentsz JO, Heijmink SW. Value of 3-T magnetic resonance imaging in local staging of prostate cancer. Top Magn Reson Imaging 2008;19:285-9.

21. Ramchandani P, Schnall MD, LiVolsi VA, Tomaszewski JE, Pollack HM. Senile amyloidosis of the seminal vesicles mimicking metastatic spread of prostatic carcinoma on MR images. AJR Am J Roentgenol 1993;161:99-100.

22. $\mathrm{Yu} \mathrm{KK}$, Hawkins RA. The prostate: Diagnostic evaluation of metastatic disease. Radiol Clin North Am 2000;38:139-57.

23. Daneshmand S, Quek ML, Stein JP, Lieskovsky G, Cai J, Pinski J, et al. Prognosis of patients with lymph node positive prostate cancer following radical prostatectomy: Long-term results. J Urol 2004;172:2252-5.

24. Greene F. Prostate. AJCC Cancer Staging Handbook. $6^{\text {th }}$ ed. New York, London: Springer; 2002. p. 309-16.

25. Le Bihan D. Diffusion/perfusion MR imaging of the brain: From structure to function. Radiology 1990;177:328-9.

26. Kim CK, Park BK, Kim B. Diffusion-weighted MRI at $3 \mathrm{~T}$ for the evaluation of prostate cancer. AJR Am J Roentgenol 2010;194:1461-9.

27. Gibbs P, Pickles MD, Turnbull LW. Diffusion imaging of the prostate at 3.0 tesla. Invest Radiol 2006;41:185-8.

28. Coakley FV, Hricak H. Radiologic anatomy of the prostate gland: A clinical approach. Radiol Clin North Am 2000;38:15-30.

29. Tamada $T$, Sone $T$, Toshimitsu S, Imai S, Jo Y, Yoshida $K$, et al. Age-related and zonal anatomical changes of apparent diffusion coefficient values in normal human prostatic tissues. J Magn Reson Imaging 2008;27:552-6.

30. Haider MA, van der Kwast TH, Tanguay J, Evans AJ, Hashmi AT, Lockwood G, et al. Combined T2-weighted and diffusion-weighted MRI for localization of prostate cancer. AJR Am J Roentgenol 2007;189:323-8

31. Kim JK, Jang YJ, Cho G. Multidisciplinary functional MR imaging for prostate cancer. Korean J Radiol 2009;10:535-51.

32. Kim CK, Choi D, Park BK, Kwon GY, Lim HK. Diffusion-weighted MR imaging for the evaluation of seminal vesicle invasion in prostate cancer: Initial results. J Magn Reson Imaging 2008;28:963-9.

33. Langer DL, van der Kwast TH, Evans AJ, Sun L, Yaffe MJ, Trachtenberg J, et al. Intermixed normal tissue within prostate cancer: Effect on MR imaging measurements of apparent diffusion coefficient and T2-sparse versus dense cancers. Radiology 2008;249:900-8.

34. Kim CK, Park BK, Lee HM. Prediction of locally recurrent prostate cancer after radiation therapy: Incremental value of 3T diffusion-weighted MRI. J Magn Reson Imaging 2009;29:391-7.

35. Hambrock T, Fütterer JJ, Huisman HJ, Hulsbergen-vandeKaa C, van Basten JP, van Oort I, et al. Thirty-two-channel coil 3T magnetic resonance-guided biopsies of prostate tumor suspicious regions identified on multimodality 3T magnetic resonance imaging: Technique and feasibility. Invest Radiol 2008;43:686-94.

36. Somford DM, Fütterer JJ, Hambrock T, Barentsz JO. Diffusion and perfusion MR imaging of the prostate. Magn Reson Imaging Clin N Am 2008;16:685-95.

37. Jackson MW, Bentel JM, Tilley WD. Vascular endothelial growth factor (VEGF) expression in prostate cancer and benign prostatic hyperplasia. J Urol 1997;157:2323-8.

38. Choi YJ, Kim JK, Kim N, Kim KW, Choi EK, Cho KS. Functional MR imaging of prostate cancer. Radiographics 2007;27:63-75.

39. Kim JK, Hong SS, Choi YJ, Park SH, Ahn H, Kim CS, et al. Wash-in rate on the basis of dynamic contrast-enhanced MRI: Usefulness for prostate cancer detection and localization. J Magn Reson Imaging 2005;22:639-46.

40. Fuchsjäger M, Shukla-Dave A, Akin O, Barentsz J, Hricak H. Prostate cancer imaging. Acta Radiol 2008;49:107-20.

41. Ocak I, Bernardo M, Metzger G, Barrett T, Pinto P, Albert PS, et al. Dynamic contrast-enhanced MRI of prostate cancer at $3 \mathrm{~T}$ : A study of pharmacokinetic parameters. AJR Am J Roentgenol 2007;189:849.

42. Jackson AS, Reinsberg SA, Sohaib SA, Charles-Edwards EM, Jhavar S, Christmas TJ, et al. Dynamic contrast-enhanced MRI for prostate cancer localization. Br J Radiol 2009;82:148-56.

43. Kayhan A, Fan X, Oommen J, Oto A. Multi-parametric MR imaging of transition zone prostate cancer: Imaging features, detection and staging. World J Radiol 2010;2:180-7.

44. Yoshizako T, Wada A, Hayashi T, Uchida K, Sumura M, Uchida N, et al. Usefulness of diffusion-weighted imaging and dynamic contrast-enhanced magnetic resonance imaging in the diagnosis of prostate transition-zone cancer. Acta Radiol 2008;49:1207-13.

45. Haider MA, Chung P, Sweet J, Toi A, Jhaveri K, Ménard C, et al. Dynamic contrast-enhanced magnetic resonance imaging for localization of recurrent prostate cancer after external beam radiotherapy. Int J Radiat Oncol Biol Phys 2008;70:425-30.

46. Cirillo S, Petracchini M, Scotti L, Gallo T, Macera A, Bona MC, et al. Endorectal magnetic resonance imaging at 1.5 Tesla to assess local recurrence following radical prostatectomy using T2-weighted and contrast-enhanced imaging. Eur Radiol 2009;19:761-9.

47. Brown TR, Kincaid BM, Ugurbil K. NMR chemical shift imaging in three dimensions. Proc Natl Acad Sci U S A 1982;79:3523-6.

48. Coakley FV, Kurhanewicz J, Qayyum A. Prostate. In: Edelman RR, Hesselink JR, Zlatkin MB, Crues JV, editors. Clinical magnetic resonance imaging. Philadelphia, PA: Elsevier; 2006. p. 2906-31.

49. Hricak H, Choyke PL, Eberhardt SC, Leibel SA, Scardino PT. Imaging prostate cancer: A multidisciplinary perspective. Radiology 2007;243:28-53.

50. Jung JA, Coakley FV, Vigneron DB, Swanson MG, Qayyum A, Weinberg V, et al. Prostate depiction at endorectal MR spectroscopic imaging: Investigation of a standardized evaluation system. Radiology 2004;233:701-8.

51. Engelhard K, Hollenbach HP, Deimling M, Kreckel M, Riedl C. Combination of signal intensity measurements of lesions in the peripheral zone of prostate with MRI and serum PSA level for differentiating benign disease from prostate cancer. Eur Radiol 2000;10:1947-53.

52. Van Dorsten FA, van der Graaf M, Engelbrecht MR, van Leenders GJ, Verhofstad A, Rijpkema M, et al. Combined quantitative dynamic contrast-enhanced MR imaging and (1)H MR spectroscopic imaging of human prostate cancer. J Magn Reson Imaging 2004;20:279-87.

53. Scheidler J, Hricak H, Vigneron DB, Yu KK, Sokolov DL, Huang LR, et al. Prostate cancer: Localization with three-dimensional proton MR spectroscopic imaging-Clinicopathologic study. Radiology 1999;213:473-80.

54. Pickett B, Kurhanewicz J, Coakley F, Shinohara K, Fein B, Roach $\mathrm{M} 3^{\text {rd }}$. Use of MRI and spectroscopy in evaluation of external beam radiotherapy for prostate cancer. Int J Radiat Oncol Biol Phys 2004;60:1047-55.

55. Harisinghani MG, Barentsz J, Hahn PF, Deserno WM, Tabatabaei S, van de $\mathrm{Kaa} \mathrm{CH}$, et al. Noninvasive detection of clinically occult lymph-node metastases in prostate cancer. N Engl J Med 2003;348:2491-9.

56. Heesakkers RA, Hövels AM, Jager GJ, van den Bosch HC, Witjes JA, Raat HP, et al. MRI with a lymph-node-specific contrast agent as an alternative to CT scan and lymph-node dissection in patients with prostate cancer: A prospective multicohort study. Lancet Oncol 2008;9:850-6.

Cite this article as: Hedgire SS, Oei TN, Mcdermott S, Cao K, Patel ZM, Harisinghani MG. Multiparametric magnetic resonance imaging of prostate cancer. Indian J Radiol Imaging 2012;22:160-9

Source of Support: Nil, Conflict of Interest: None declared. 